\title{
APLICACIÓN DE UN PROGRAMA DE EVALUACIÓN E INTERVENCIÓN SOBRE LA DISORTOGRAFÍA
}

\section{IMPLEMENTATION OF A PROGRAMME TO EVALUATE AND INTERVENE ON DISORTOGRAPHY}

\author{
Rocío Lavigne*, Juan F. Romero** y Gemma Rodríguez*** \\ Universidad de Málaga
}

\section{RESUMEN}

La presente investigación forma parte de un amplio Programa de Evaluación y Mejora del Rendimiento Académico en alumnos de Enseñanza Secundaria Obligatoria (ESO). Se presenta el estudio de un Programa de Evaluación e Intervención sobre las dificultades encontradas en el lenguaje escrito, en concreto en la Ortografía, en alumnos del primer ciclo de ESO, con objeto de mejorar la ortografía y finalmente su rendimiento académico. La evaluación puso de manifiesto el elevado número de errores que los alumnos cometían tanto de ortografía natural como arbitraria, que afectaba a su rendimiento académico en todas las áreas. La aplicación de dos Programas de Mejora de la Ortografía Natural y Arbitraria mostró que la inclusión en el diseño curricular y el plan de aula de actividades específicas como la realizada pueden tener efectos relevantes en la escritura y en el rendimiento escolar de los alumnos.

Palabras clave: Disortografía, Evaluación, Intervención.

\section{ABSTRACT}

The present study is part of a wide Evaluation and Achievement Program of Scholar Performance with learners of the Enseñanza Secundaria Obligaroria (E.S.O.) -from 12 to 16 years. The evaluation and Intervention Program of disabilities in written language, making special emphasis in ortography,

\footnotetext{
* Rocío Lavigne Cerván: Maestra y Psicopedagoga. Profesora asociada en el Departamento de Psicología Evolutiva de la Facultad de Ciencias de la Educación de la Universidad de Málaga y Orientadora en un Centro Concertado de Educación Infantil y Primaria. Investigadora del Grupo de Investigación PAI HUM-347.

** Juan F. Romero Pérez: Profesor titular de Psicología Evolutiva y de la Educación en la Universidad de Málaga, con amplia experiencia docente e investigadora en el campo de las dificultades en el aprendizaje. Responsable del Grupo de Investigación del PAI HUM-347.

*** Gemma Rodriguez Infante: Licenciada en Psicopedagogía. Profesora Asociada en el Departamento de Psicología Evolutiva de la Facultad de Ciencias de la Educación de la Universidad de Málaga. E investigadora del Grupo de Investigación PAI HUM-347.
} 
was conducted on a group of students in the First Cycle of E.S.O.. The aim of the present study is the improvement of the learners'ortography and, consequently, their Scholar Performance. The evaluation revealed the large number of mistakes that were made by the students in natural and arbitrary ortography, which affected their Scholar Performance in all areas. The application of two Programs for the Achievement of Natural and Arbitrary Ortography failed to show evidences of the improvement of the students in writing and in Scholar Performance if these specific activities were included in the $\mathrm{Cu}$ rriculum Design and in the classroom plan.

Key words: Disortography, Evaluation, Intervention.

\section{Introducción}

La presente investigación forma parte de un amplio Programa de Evaluación y Mejora del Rendimiento Académico en alumnos de Enseñanza Secundaria Obligatoria (ESO). Se presenta el estudio de un Programa de Evaluación e Intervención sobre las dificultades encontradas en el lenguaje escrito, en concreto en la Ortografía, en alumnos del primer ciclo de ESO, con objeto de mejorar la ortografía y finalmente su rendimiento académico.

La escritura es un término que puede ser empleado de forma ambigua, se utiliza tanto para denominar la actividad proceso de escritura como para designar el producto de dicha actividad. Por ello, en ocasiones se utiliza el término para referirse a los aspectos caligráficos y ortográficos de la escritura, y otras se utiliza para referirse a aspectos expresivos y comunicativos mediante la escritura espontánea. Según Sánchez (1999), para dominar el lenguaje escrito hay que superar dos retos que tienen una naturaleza muy distinta. El primero se trata en adquirir las habilidades que permiten pasar de la fonología de las palabras a la ortografía. El segundo, supone llegar a utilizar estas habilidades para comunicarnos con los demás.

a) ¿Qué hacemos cuando escribimos un texto de forma espontánea?

a.1. Planificación del mensaje

a.2. Construcción de las Estructuras Sintácticas

a.3. Procesos Léxicos o de Recuperación de la Palabra

a.4. Procesos Motores

b) ¿Qué hacemos cuándo escribimos un dictado?

b.1. Procesos Léxicos o de Recuperación de la Palabra: Consiste en la elección de las palabras que se incorporarán dentro de la estructura sintáctica seleccionada. Los mecanismos que nos permiten recuperar la forma de la palabra en la escritura son los siguientes:

- Recuperación Ortográfica de la forma de la palabra, directa o visual (ver esquema 1)

- Recuperación Fonológica Indirecta de la forma de la palabra (ver esquema 2)

Según Cuetos (1991) y García, J.N. (1995), cuando las palabras que tenemos que escribir al dictado son cortas, familiares y conocidas, lo más frecuente es que se haga uso de la ruta visual, mediante la recuperación ortográfica de la palabra. Así al escribir una palabra dictada, funcionamos de la siguiente forma: 


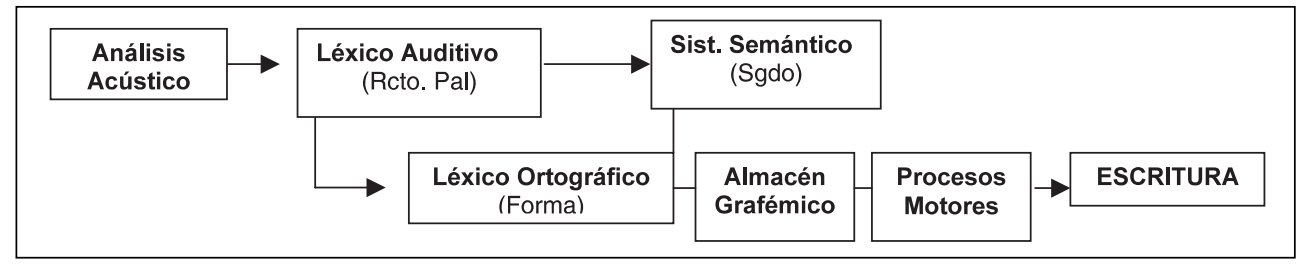

\section{ESQUEMA 1.}

En el caso de que se trate de palabras desconocidas o pseudopalabras, se hará uso de la ruta fonológica, tal y como vemos en el siguiente esquema:

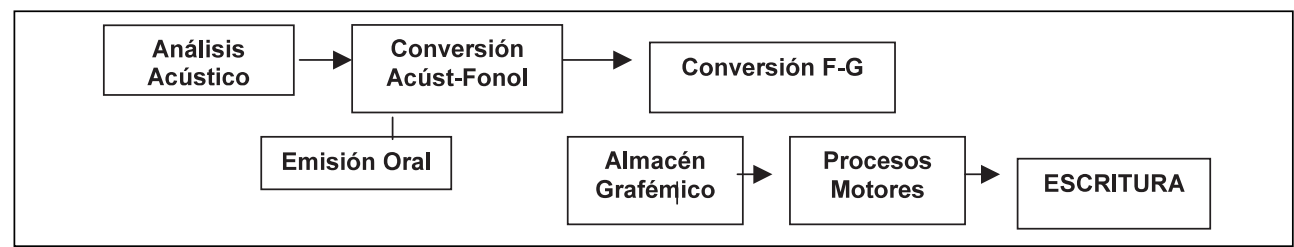

ESQUEMA 2.

b.2. Procesos Motores: para representar la palabra sobre cualquier superficie, se tienen que recuperar los alógrafos oportunos y transformarlos en movimiento. Para ello, hay que acudir al almacén alográfico en la M.L.P. Los movimientos relativos a cada alógrafo están también allí, esta información debe pasar a la M.C.P., donde se especificarán la dirección, tamaño de las letras, etc. Lo único que falta es representar el grafema sobre el papel, mediante los patrones motores correspondientes, que en este caso se trata de los movimientos de los dedos y la muñeca.

c) ¿Qué hacemos cuándo escribimos un copiado?

c.1. Procesos Léxicos: en el caso de que el sujeto sepa leer.

c.2. Procesos Motores

En el siguiente cuadro, podemos apreciar de modo resumido los diferentes procesos cognitivos que se ponen en juego en cada tipo de escritura: escritura espontánea, dictado y copia.

\begin{tabular}{|l|c|c|c|}
\hline \multicolumn{1}{|c|}{ Procesos Cognitivos } & Escritura Espontánea & Dictado & Copia \\
\hline Planificación & $\mathrm{X}$ & & \\
\hline Construcción de Estructuras Sintácticas & $\mathrm{X}$ & & \\
\hline Procesos Léxicos & $\mathrm{X}$ & $\mathrm{X}$ & $\mathrm{X}^{1}$ \\
\hline Procesos Motores & $\mathrm{X}$ & $\mathrm{X}$ & $\mathrm{X}$ \\
\hline
\end{tabular}

1 En el caso de que el sujeto sepa llevar a cabo la conversión G-F - F-G, si no es así los únicos procesos que se activan son los motores. 


\section{d) Variables implicadas en la Escritura}

Variables psicológicas del sujeto que intervienen directamente en el proceso escritor y que en ocasiones son las causantes de las DAE: La atención sostenida y selectiva; La memoria a corto plazo, la memoria a largo plazo y la memoria de trabajo; La percepción y la discriminación; La lateralidad; La coordinación visomotriz; La conciencia fonológica; El léxico fonológico.

\section{Las Dificultades en el Aprendizaje de la Escritura. La Disortografía}

Las Dificultades en el Aprendizaje de la Escritura (DAE), hacen referencia a las desadaptaciones que se presentan en el curso de procesos de Enseñanza y Aprendizaje que tienen como objetivo último el aprendizaje de la Escritura. Generalmente, las DAE aparecen asociadas con las Dificultades en el Aprendizaje de la Lectura (DAL), porque constituyen aprendizajes interactivos.

Dentro de las DAE, nos encontramos con la DISORTOGRAFÍA: se trata de una inhabilidad en la recuperación de la forma de las palabras relacionada con errores fonológicos en la conversión fonema-grafema y/o con errores en las reglas ortográficas y de puntuación. Existen dos tipos de disortografías:

a) Disortografías Naturales: Son aquellas que tienen que ver con el desarrollo fono articulatorio y con el desarrollo de la conciencia fonológica y de las reglas de conversión fonema-grafema.

Entendemos por errores causados por un mal desarrollo fono-articulatorio, aquellos errores cometidos en la Ortografía Natural $(\mathrm{ON})$, por la incorrecta pronunciación de los fonemas que se emiten, y por la escritura de los grafemas, tal y como se pronuncian los fonemas. Y por conciencia fonológica, el conocimiento de la estructura interna del lenguaje. Los errores más frecuentes en este tipo de disortografía son los siguientes: Sustituciones, Omisiones, Rotaciones, Inversiones, Adiciones, Uniones y Fragmentaciones.

b) Disortografía Arbitrarias: Aquí la escritura fonológica es correcta pero no lo es ortográficamente ya que no se utilizan las normas ortográficas. Las causas de la disortografía arbitraria pueden ser; una mala instrucción; una inexistente o errónea representación mental de la palabra; y/o una representación mental de la palabra que no se consulta, limitándose a hacer la conversión f-g.

A los errores relacionados con esta disortografía se le denominan errores de OA, es decir, errores relacionados con el conocimiento y uso de las reglas ortográficas. Los más comunes son: Acentuación, puntuación, B/V, R/RR, Ll/Y, H omitida, H adicional, $\mathrm{M}$ antes de B/P, G/J, GU/G, C/QU/K, Mayúsculas y minúsculas. 


\section{Parte práctica}

\section{Metodología}

\section{Objetivos}

Objetivo general: elaboración y aplicación de dos Programas de Mejora de la Ortografía, natural (PON) y/o arbitraria (POA), dirigidos a un grupo de alumnos del primer ciclo de ESO que cometen errores ortográficos.

Objetivos específicos: a) Mejorar el desarrollo fonológico y los conocimientos sobre las reglas de conversión fonema-grafema de los alumnos intervenidos mediante el PON; b) Conseguir que los alumnos intervenidos mediante el Programa de Mejora de la Ortografía Arbitraria, sean capaces de dominar las reglas ortográficas.

\section{Muestra}

Para la fase de evaluación participaron un total de 123 alumnos de $1^{\circ}$ y $2^{\circ}$ de ESO, (Véase tabla 1), de un instituto de ESO privado concertado, de aproximadamente 700 alumnos, situado en una zona céntrica de Málaga capital.

TABLA 1: Distribución de los sujetos que han sido evaluados.

\begin{tabular}{|c|c|c|c|}
\hline CURSO & GRUPO & $\begin{array}{c}\text { Alumnos } \\
\text { Evaluados }\end{array}$ & TOTAL \\
\hline \multirow{2}{*}{$1^{\circ}$ ESO } & A & 26 & 54 \\
& B & 28 & 69 \\
\hline \multirow{2}{*}{$2^{\circ}$ ESO } & $\mathrm{A}$ & 35 & 69 \\
& $\mathrm{~B}$ & 34 & 123 \\
\cline { 3 - 4 }
\end{tabular}

Participaron en los programas de mejora aquellos alumnos que además de puntuar por encima de la mediana, tenían el visto bueno por sus profesores. Así, los alumnos que en $1^{\circ}$ de ESO tuvieran más de 9 errores y los que de $2^{\circ}$ ESO tuviesen más de 18 , serían aquellos a los que se aplicarán los programas de mejora de la ortografía. (Véase tabla 2).

TABLA 2: Distribución de los sujetos que han sido seleccionados e intervenidos.

\begin{tabular}{|c|c|c|c|}
\hline CURSO & GRUPO & $\begin{array}{c}\text { Alumnos } \\
\text { Intervenidos }\end{array}$ & TOTAL \\
\hline \multirow{2}{*}{$1^{\circ} \mathrm{ESO}$} & $\mathrm{A}$ & 9 & 15 \\
& $\mathrm{~B}$ & 6 & \\
\hline \multirow{2}{*}{$2^{\circ} \mathrm{ESO}$} & $\mathrm{A}$ & 8 & 15 \\
& $\mathrm{~B}$ & 7 & 30 \\
\cline { 2 - 4 }
\end{tabular}




\section{Hipótesis}

- Primera Hipótesis. Se espera que la aplicación del PON, suponga una mejora en la Ortografía Natural de todo el grupo intervenido.

- Segunda Hipótesis. Se espera que la aplicación del PON, suponga una mejora en la Ortografía Total (ON y OA) de todo el grupo intervenido.

- Tercera Hipótesis. Se espera que la aplicación del POA, suponga una mejora en la Ortografía Arbitraria de todo el grupo intervenido.

- Cuarta Hipótesis. Se espera que la aplicación del POA, suponga una mejora en la Ortografía Total (OA y ON) de todo el grupo intervenido.

- Quinta Hipótesis. Se espera que la aplicación de ambos programas provoquen mejoras en el rendimiento escolar, en el área de Lengua Castellana y Literatura.

\section{Variables}

Variables Dependientes: el número de errores ortográficos que cometen los alumnos, tanto errores ortográficos naturales como arbitrarios, y el rendimiento académico de los alumnos definidos en términos del área de Lengua Castellana.

Variables Independientes: todas aquellas que se trabajan directamente a través del PON y del POA. En el PON hablamos de «desarrollo fonoarticulatorio» y «desarrollo de la conciencia fonológica», en el POA de «aprendizaje de reglas ortográficas», y en ambos de «Metacognición».

\section{Instrumentos de medida}

Para la evaluación, se utilizaron dos dictados, (uno para $1^{\circ}$ y otro para $2^{\circ}$ de ESO), de una extensión de aproximadamente 15 renglones.

- Criterios de corrección: un punto por error cometido, de entre los siguientes: (los errores fueron anotados en unas hojas de registro).

- Errores de Ortografía Natural, es decir, errores relacionados con los conocimientos fonológicos (articulación de sonidos del habla, conciencia fonológica, entre otros) y con el conocimiento de las reglas de conversión f-g.

- Errores de Ortografía Arbitraria, es decir, errores relacionados con el conocimiento y uso de las reglas ortográficas.

\section{Diseño}

El diseño utilizado para el desarrollo del Programa es del tipo ABA, longitudinal. En la fase «A», que tuvo una duración de dos semanas, se aplicó el pretest a todos los alumnos. En la fase «B», con una duración de cuatro meses, a razón de una sesión por semana de una hora de duración, se aplicaron los Programas (PON y POA), a aquellos alumnos que en el pretest, obtuvieron una puntuación por encima de la mediana. Y por último, se repitió la 
fase «A», en la que se aplicó el post-test a todos los alumnos, tanto a los intervenidos como a los que no participaron en la intervención.

\section{Componentes del PON}

- Desarrollo fono-articulatorio.

- Desarrollo de la conciencia fonológica.

- Meta-cognición.

\section{Desarrollo del PON}

Cada una de las sesiones que forman parte del programa se ha aplicado siguiendo la secuencia que a continuación se muestra, en grupos reducidos de alumnos, durante diez sesiones, a razón de una sesión por semana.

1. Se establecía el propósito particular de cada sesión.

2. Se revisaban las lecturas realizadas durante la semana y se proponían otras para la siguiente.

3. Se leían textos seleccionados que contenían aspectos sobre los que se iba a trabajar.

4. Realización de las tareas correspondientes, dependiendo de los objetivos que se perseguían en cada sesión. De este modo se realizaron actividades relacionadas con:

- Segmentación de sílabas y fonemas; Segmentación de sílabas y grafemas; Correspondencias f-g y g-f; Tareas de adición y supresión de fonemas y de grupos de fonemas (variado su posición en la palabra); Tareas de adición y supresión de grafemas y grupos de grafemas (variando su posición en la palabra); Tareas con palabras que se escriben de forma diferente a como se pronuncian; Tareas con palabras desconocidas; Escritura de pseudopalabras; etc.

El procedimiento en cada sesión se ajusta al contenido y objetivos específicos, así se realizaban dictados, escritura espontánea, etcétera.

5. Autoevaluación y comentario de los resultados.

6. Meta-cognición: mediante auto-preguntas dirigidas se intentaba mejorar el desarrollo meta-cognitivo de los alumnos, es decir, la conciencia del proceso realizado y de las variables personales implicadas, sus atribuciones y expectativas de control sobre el aprendizaje y los resultados.

\section{Componentes del POA}

- Conocimiento de las reglas Ortográficas.

- Meta-cognición. 


\section{Desarrollo del POA}

El programa se aplicará siguiendo la secuencia que a continuación se muestra en grupos reducidos de alumnos en diez sesiones, a razón de una sesión por semana:

1. Establecer el propósito general del Programa, (esto sólo en la primera sesión), y particular de cada sesión.

2. Revisión de las lecturas realizadas durante la semana y propuestas para la semana siguiente.

3. Lectura de textos seleccionados que contengan aspectos sobre los que se va a trabajar.

4. Desarrollo de las tareas:

- Actividades de memoria visual inmediata y de atención sostenida (sopas de letras, identificación de modelos, etc.); Normas de uso de «b/v», omisión de «h», adición de «h», de acentuación; de puntuación; mayúsculas y minúsculas; etc.

El procedimiento en cada sesión se ajusta al contenido y objetivos específicos, así se realizan dictados, escritura espontánea, etcétera.

5. Autoevaluación y comentario de los resultados.

6. Meta-cognición: mediante auto-preguntas dirigidas se intenta mejorar el desarrollo meta-cognitivo de los alumnos, es decir, la conciencia del proceso realizado y de las variables personales implicadas, sus atribuciones y expectativas de control sobre el aprendizaje y los resultados.

\section{Resultados}

\section{Resultados del Pretest en la Ortografía Natural (ON)}

Resultados del Pretest de la ON de toda la muestra

Los resultados de la evaluación que se realizó en Noviembre de 1999, ponen de manifiesto, que todos los alumnos cometen una gran cantidad de errores ortográficos naturales, cuando este tipo de errores debería estar totalmente erradicado a estas edades (véase tabla 3).

TABLA 3: Resultados de los errores del Pretest en la ON de toda la muestra.

\begin{tabular}{|c|c|c|c|c|c|c|c|c|c|c|c|c|c|c|c|c|c|}
\hline \multicolumn{10}{|c|}{$\mathbf{1}^{\circ}$ DE ESO } & \multicolumn{10}{c|}{$\mathbf{2}^{\circ}$ DE ESO } \\
\hline \multicolumn{3}{|c|}{ A } & \multicolumn{3}{|c|}{ B } & \multicolumn{3}{c|}{ TOTAL } & \multicolumn{3}{c|}{ A } & \multicolumn{3}{c|}{ B } & \multicolumn{3}{c|}{ TOTAL } \\
\hline X & DT & N & X & DT & N & X & DT & N & X & DT & N & X & DT & N & X & DT & N \\
\hline 6,42 & 7,81 & 26 & 4,96 & 6,53 & 28 & 5,67 & 7,15 & 54 & 6,94 & 8,61 & 35 & 5,38 & 7,4 & 34 & 6,17 & 8,02 & 69 \\
\hline
\end{tabular}

Resultados del Pretest de la ON de los alumnos intervenidos

El PON fue aplicado a los alumnos de $1^{\circ}$ de ESO, cuya puntuación en el número total de errores ortográficos estuviese por encima de la mediana, que en $1^{\circ}$ fue de «9». Si observa- 
mos la gráfica, (véase tabla 4), vemos cómo en $2^{\circ} \mathrm{A}$, la media es mayor que la de $1^{\circ} \mathrm{A}$. Sin embargo, a los alumnos de $1^{\circ} \mathrm{A}$ se les incluyó en el PON, debido a que, el número de errores naturales no era despreciable, y además, como veremos posteriormente, era mayor que el número de errores arbitrarios. Por otro lado, los alumnos de $2^{\circ}$ tuvieron muchos más errores relacionados con la Ortografía Arbitraria que con la Natural y fue a ellos a los que se les aplicó el POA.

TABLA 4: Resultados de los errores del Pretest en la ON de los alumnos intervenidos.

\begin{tabular}{|c|c|c|c|c|c|c|c|c|c|c|c|c|c|c|c|c|c|}
\hline \multicolumn{9}{|c|}{$1^{\circ}$ DE ESO } & \multicolumn{1}{c|}{$2^{\circ}$ DE ESO } \\
\hline \multicolumn{3}{|c|}{ A } & \multicolumn{3}{|c|}{ B } & \multicolumn{3}{c|}{ TOTAL } & \multicolumn{3}{c|}{ A } & \multicolumn{3}{c|}{ B } & \multicolumn{3}{c|}{ TOTAL } \\
\hline $\mathrm{X}$ & $\mathrm{DT}$ & $\mathrm{N}$ & $\mathrm{X}$ & $\mathrm{DT}$ & $\mathrm{N}$ & $\mathrm{X}$ & $\mathrm{DT}$ & $\mathrm{N}$ & $\mathrm{X}$ & $\mathrm{DT}$ & $\mathrm{N}$ & $\mathrm{X}$ & $\mathrm{DT}$ & $\mathrm{N}$ & $\mathrm{X}$ & $\mathrm{DT}$ & $\mathrm{N}$ \\
\hline 6,22 & 2,81 & 9 & 13,5 & 10 & 6 & 9,13 & 4,97 & 15 & 9,25 & 5,12 & 8 & 7,71 & 3,03 & 7 & 8,53 & 4,20 & 15 \\
\hline
\end{tabular}

\section{Resultados del Pretest en la Ortografía Arbitraria (OA)}

Resultados del Pretest de la OA de toda la muestra

Los resultados de la evaluación que se realizó en Noviembre de 1999, ponen de manifiesto, que todos los alumnos cometen una gran cantidad de errores ortográficos arbitrarios, (véase tabla 5).

TABLA 5: Resultados de los errores del Pretest en la OA de toda la muestra.

\begin{tabular}{|c|c|c|c|c|c|c|c|c|c|c|c|c|c|c|c|c|c|}
\hline \multicolumn{9}{|c|}{$1^{\circ}$ DE ESO } & \multicolumn{9}{c|}{$2^{\circ}$ DE ESO } \\
\hline \multicolumn{3}{|c|}{ A } & \multicolumn{3}{|c|}{ B } & \multicolumn{3}{|c|}{ TOTAL } & \multicolumn{3}{|c|}{ A } & \multicolumn{3}{c|}{ B } & \multicolumn{3}{c|}{ TOTAL } \\
\hline X & DT & N & X & DT & N & X & DT & N & X & DT & N & X & DT & N & X & DT & N \\
\hline 5,85 & 3,62 & 26 & 6,54 & 4,99 & 28 & 6,2 & 4,36 & 54 & 14,8 & 5,75 & 35 & 13,3 & 5,75 & 34 & 14,0 & 5,76 & 69 \\
\hline
\end{tabular}

Resultados del Pretest de la OA de los alumnos intervenidos

El POA fue aplicado a los alumnos de $2^{\circ}$ de ESO, cuya puntuación en el número total de errores ortográficos estuviese por encima de la mediana que fue de 18. Se aplicó este Programa con $2^{\circ}$ de ESO, debido a que al comparar las medias de los sujetos, tanto de $1^{\circ}$ como de $2^{\circ}$ que iban a ser intervenidos, se pudo comprobar (ver tabla 6) cómo la media de los errores arbitrarios en $1^{\circ}$ fue menor que la de $2^{\circ}$.

TABLA 6: Resultados de los errores del Pretest en la OA de los alumnos intervenidos.

\begin{tabular}{|c|c|c|c|c|c|c|c|c|c|c|c|c|c|c|c|c|c|}
\hline \multicolumn{9}{|c|}{$1^{\circ}$ DE ESO } & \multicolumn{1}{c|}{$2^{\circ}$ DE ESO } \\
\hline \multicolumn{3}{|c|}{ A } & \multicolumn{3}{|c|}{ B } & \multicolumn{3}{|c|}{ TOTAL } & \multicolumn{3}{c|}{ A } & \multicolumn{4}{c|}{ B } & \multicolumn{3}{c|}{ TOTAL } \\
\hline $\mathrm{X}$ & $\mathrm{DT}$ & $\mathrm{N}$ & $\mathrm{X}$ & $\mathrm{DT}$ & $\mathrm{N}$ & $\mathrm{X}$ & $\mathrm{DT}$ & $\mathrm{N}$ & $\mathrm{X}$ & $\mathrm{DT}$ & $\mathrm{N}$ & $\mathrm{X}$ & $\mathrm{DT}$ & $\mathrm{N}$ & $\mathrm{X}$ & $\mathrm{DT}$ & $\mathrm{N}$ \\
\hline 7,77 & 3,66 & 9 & 13,8 & 4,62 & 6 & 9,13 & 7,34 & 15 & 16,1 & 4,08 & 8 & 18,7 & 4,19 & 7 & 17,3 & 4,20 & 15 \\
\hline
\end{tabular}




\section{Resultados del Postest}

\section{Resultados del Postest en la ON}

Resultados del Postest de la ON de los alumnos intervenidos

Los resultados del postest que se realizó en Junio del 2000, ponen de manifiesto, que muchos de los alumnos intervenidos siguen cometiendo errores ortográficos naturales, pero el número de estos ha descendido, con respecto a los resultados obtenidos en el pretest (Tabla 7).

TABLA 7: Resultados de los errores del Postest en la ON de los alumnos intervenidos.

\begin{tabular}{|c|c|c|c|c|c|c|c|c|c|c|c|c|c|c|c|c|c|}
\hline \multicolumn{9}{|c|}{$1^{\circ}$ DE ESO } & \multicolumn{9}{|c|}{$2^{\circ}$ DE ESO } \\
\hline \multicolumn{3}{|c|}{ A } & \multicolumn{3}{|c|}{ B } & \multicolumn{3}{|c|}{ TOTAL } & \multicolumn{3}{|c|}{ A } & \multicolumn{3}{|c|}{ B } & \multicolumn{3}{|c|}{ TOTAL } \\
\hline $\mathrm{X}$ & DT & $\mathrm{N}$ & $X$ & DT & $\mathrm{N}$ & $\mathrm{X}$ & DT & $\mathrm{N}$ & $\mathrm{X}$ & DT & $\mathrm{N}$ & $\mathrm{X}$ & DT & $\mathrm{N}$ & X & DT & $\mathrm{N}$ \\
\hline 5,67 & 3,24 & 9 & 5,5 & 5,13 & 6 & 5,6 & 3,92 & 15 & 3,25 & 3,45 & 8 & 1 & 1,41 & 7 & 2,2 & 2,86 & 15 \\
\hline
\end{tabular}

\section{Resultados del Postest en la $O A$}

Resultados del Postest de la OA de los alumnos intervenidos

Los resultados del post-test que se realizó en Junio del 2000, (ver tabla 8), ponen de manifiesto que los alumnos intervenidos siguen cometiendo errores ortográficos arbitrarios, pero el número de estos ha descendido, con respecto a los resultados obtenidos en el pretest.

TABLA 8: Resultados de los errores del Postest en la OA de los alumnos intervenidos.

\begin{tabular}{|c|c|c|c|c|c|c|c|c|c|c|c|c|c|c|c|c|c|}
\hline \multicolumn{9}{|c|}{$1^{\circ}$ DE ESO } & \multicolumn{1}{c|}{$2^{\circ}$ DE ESO } \\
\hline \multicolumn{3}{|c|}{ A } & \multicolumn{3}{|c|}{ B } & \multicolumn{3}{|c|}{ TOTAL } & \multicolumn{3}{|c|}{ A } & \multicolumn{4}{c|}{ B } & \multicolumn{3}{c|}{ TOTAL } \\
\hline X & DT & N & X & DT & N & X & DT & N & X & DT & N & X & DT & N & X & DT & N \\
\hline 2,78 & 1,56 & 9 & 5,5 & 4,09 & 6 & 3,87 & 3,04 & 15 & 4,5 & 3,96 & 8 & 6,71 & 3,09 & 7 & 5,53 & 3,64 & 15 \\
\hline
\end{tabular}

\section{Comparación entre el Pretest y el Postest}

Como podemos observar, (ver tabla 9 y gráfica 1), los alumnos intervenidos con el PON ( $\left.1^{\circ} \mathrm{ESO}\right)$, han mejorado significativamente el número de errores en la ortografía natural. Así mismo, a pesar de que sólo fueron intervenidos en este tipo de ortografía, también el número de errores en la ortografía arbitraria ha mejorado de forma significativa. De este modo, la ortografía total (OA y $\mathrm{ON}$ ) ha experimentado un descenso significativo en lo que respecta al número de errores. 
TABLA 9: Comparación de medias de los alumnos intervenidos con el PON (1 ${ }^{\circ}$ ESO).

\begin{tabular}{|c|c|c|c|}
\hline & Media Inicial Media final & $\mathbf{T}$ & Significatividad (bilateral) \\
\hline O.N. & $\begin{array}{r}9.13 \\
5.60\end{array}$ & 2.34 & $0.034^{*}$ \\
\hline O.A. & $\begin{array}{r}10.20 \\
3.87\end{array}$ & 7.27 & $0.000^{* * *}$ \\
\hline O.T. (ON + OA) & $\begin{array}{r}19.33 \\
9.46\end{array}$ & 5.39 & $0.000^{* * *}$ \\
\hline $\mathbf{N}$ & \multicolumn{3}{|c}{15} \\
\hline
\end{tabular}

*p. $0.05 \quad * *$ p. $0.01 \quad * * *$ p. 0.001

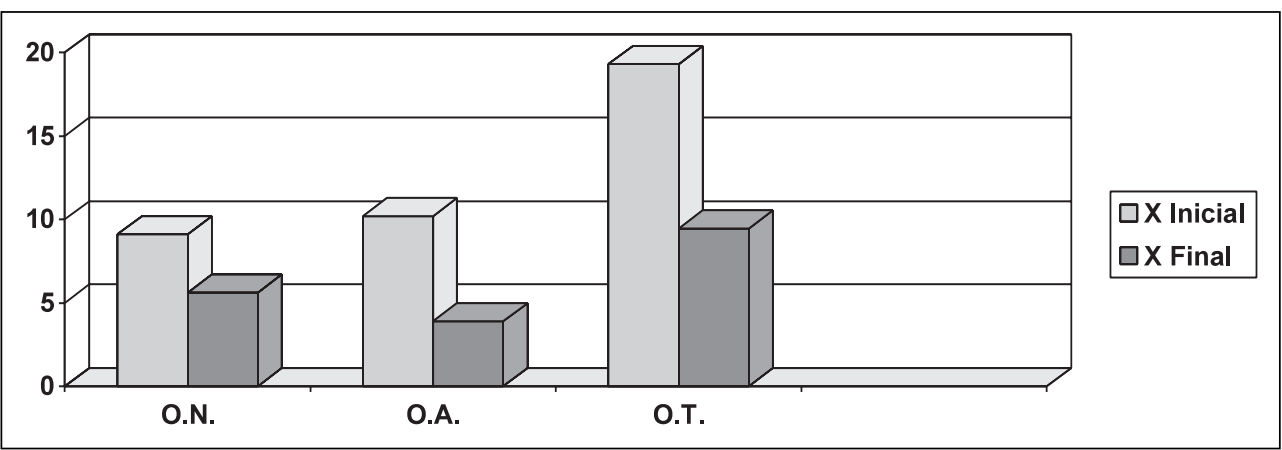

GRÁFICO 1.

Comparación de medias de los alumnos intervenidos con el PON (1 ESO).

Por otro lado, en el grupo de alumnos intervenidos con el POA, ha pasado algo similar. El número de errores en $\mathrm{OA}$, ha mejorado significativamente, así como, el número de errores relacionados con la ON y por consiguiente con la OT (OA y ON). (Véase tabla 10 y gráfica 2).

TABLA 10: Comparación de medias de los alumnos intervenidos con el POA ( $2^{\circ}$ ESO).

\begin{tabular}{|c|c|c|c|}
\hline & Media Inicial Media final & $\mathbf{T}$ & Significatividad (bilateral) \\
\hline O.A. & $\begin{array}{r}17.33 \\
5.53\end{array}$ & 8.093 & $0.000^{* * *}$ \\
\hline O.N. & $\begin{array}{r}8.53 \\
2.20\end{array}$ & 8.40 & $0.000^{* * *}$ \\
\hline O.T. (ON + OA) & $\begin{array}{r}26.53 \\
7.73\end{array}$ & 10.65 & $0.000^{* * *}$ \\
\hline $\mathbf{N}$ & \multicolumn{3}{|c|}{15} \\
\hline
\end{tabular}

*p. $0.05 \quad * *$ p. $0.01 \quad * * *$ p. 0.001 


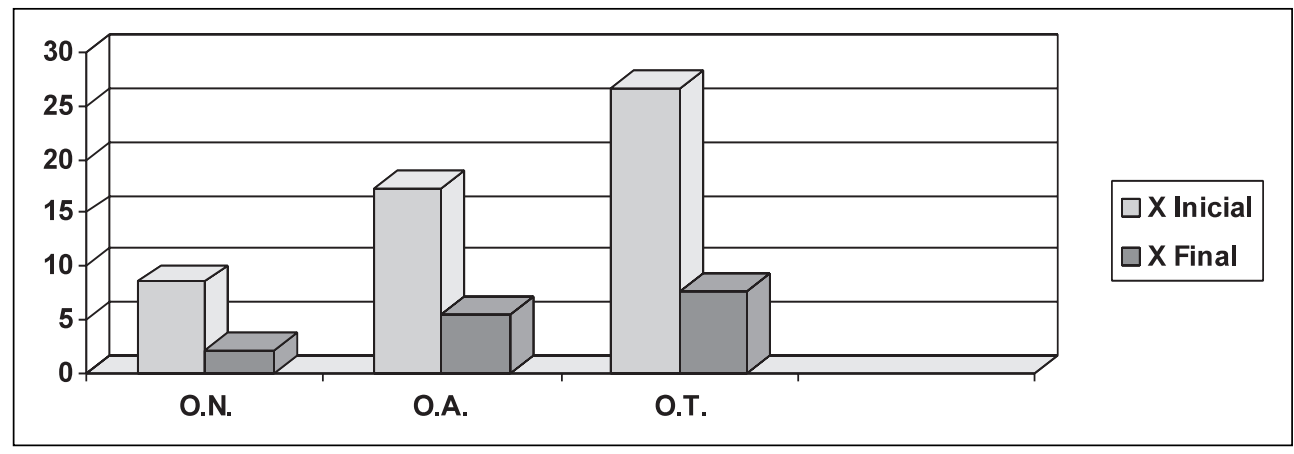

\section{GRÁFICO 2.}

Comparación de medias de los alumnos intervenidos con el POA (2 ESO).

Así vemos, que a pesar de que cada programa tiene unos objetivos diferentes, posee aspectos en común, como el incremento de la tarea lectora y el desarrollo de la metacognición, que influyen tanto para la mejora de la ON como de la OA. Estos aspectos comunes han hecho que el número de errores en la Ortografía Total (ON y OA), hayan mejorado de forma significativa.

Por otro lado para comprobar si la aplicación de los programas ha provocado mejoras en el rendimiento escolar, en el área de lengua castellana y literatura, hemos calculado la nota media de los alumnos intervenidos antes y después de la aplicación del programa ${ }^{2}$, obteniéndose que las notas de los alumnos han mejorado pero no de forma significativa. (Véase tabla 11). Esto es debido a que en la evaluación de esta materia, se tienen en cuenta otras variables, además de la ortografía, que no han sido trabajadas en nuestros programas.

TABLA 11: Comparación de las notas medias antes y después de la aplicación del PON y POA, en los alumnos intervenidos.

\begin{tabular}{|c|c|c|c|}
\hline & $\begin{array}{c}\text { Nota Media Antes } \\
\text { Nota Media Después }\end{array}$ & T & Significatividad (bilateral) \\
\hline $\mathbf{1}^{\mathbf{0}}$ ESO (PON) $(\mathbf{N}=\mathbf{1 5})$ & $\begin{array}{l}4.46 \\
4.66\end{array}$ & -1.871 & 0.082 \\
\hline $\mathbf{2}^{\circ}$ ESO (POA) $(\mathbf{N}=\mathbf{1 5})$ & $\begin{array}{l}5.00 \\
5.13\end{array}$ & -0.695 & 0.499 \\
\hline
\end{tabular}

*p. $0.05 * *$ p. $0.01 \quad * * *$ p. 0.001

\section{Discusión}

A la luz de los resultados obtenidos y con el objetivo de comprobar si se cumplen o no las hipótesis planteadas (véase apartado 2.1.3.), podemos concluir que:

2 Considerando los siguientes criterios: Insuficiente $=4 ;$ Suficiente $=5 ;$ Bien $=6 ;$ Notable $=7$;

Sobresaliente $=9$ 
a) Con respecto a la primera hipótesis, rechazamos la Ho, por lo cual demostramos que la aplicación del PON, ha supuesto una mejora significativa en la $\mathrm{ON}$ del grupo intervenido.

b) Con respecto a la segunda hipótesis, rechazamos la Ho, demostrando de este modo que la aplicación del PON, ha supuesto una mejora significativa en la Ortografía Total (ON y OA) del grupo intervenido.

c) En relación con la tercera hipótesis, rechazamos la Ho, con ello se demuestra que la aplicación del POA, ha supuesto una mejora significativa en la OA de todo el grupo intervenido.

d) Con respecto a la cuarta hipótesis, rechazamos la Ho, demostrando así que la aplicación del POA, ha supuesto también una mejora significativa en la Ortografía Total (OA y ON) del grupo intervenido.

e) Por último y en relación con la quinta hipótesis, no rechazamos la Ho, por lo que la aplicación de los programas de mejora de la ortografía no han provocado mejoras significativas en el rendimiento escolar, en el área de Lengua Castellana y Literatura.

Como hemos podido comprobar tras analizar los resultados, el número de errores ortográficos no ha desaparecido totalmente, aunque sí ha descendido de forma significativa. Esto es debido a que en el pretest los alumnos demostraron tener un elevadísimo número de faltas ortográficas. Además, la duración de los programas, por cuestiones organizativas del centro donde se llevó a cabo la investigación, ha resultado ser relativamente corta, lo que ha perjudicado a los sujetos intervenidos.

Si se compara este estudio con el de Mesanza, (2000), que aunque no es igual, es similar, podemos comprobar cómo hay coincidencias en cuanto a la cantidad de errores ortográficos encontrados en los alumnos de secundaria. Más concretamente, en lo que respecta a los errores ortográficos arbitrarios, coincidimos con este autor en que los errores de acentuación son los más abundantes.

Por otro lado, en la evaluación realizada por el Instituto Nacional de Calidad y Evaluación (INCE) del Ministerio de Educación, en el año 2000, también se señala, al igual que en nuestra evaluación, que los alumnos de la ESO, cometen un elevado número de errores ortográficos.

\section{Conclusiones}

Resulta preocupante cómo en el primer ciclo de la Secundaria aún se siguen cometiendo errores ortográficos y no sólo arbitrarios sino también naturales, cuando este tipo de errores deberían de estar totalmente erradicados en la primaria.

¿Qué está sucediendo entonces?, es la pregunta que nos hacemos muchos de los profesionales que trabajamos en este campo. Lo que está pasando es que los alumnos no adquieren los pilares fundamentales de la educación (lectura y escritura) en los niveles donde se les es exigido, y van subiendo de nivel educativo sin dominar la lectoescritura. De este modo, comienzan a tener dificultades para comprender lo que leen, para expresar sus ideas mediante mensajes escritos, para escribir sin cometer errores ortográficos, ..., debido a esto se desmotivan, y disminuyen su actividad lectoescritora, ya que no les resulta gratificante, 
ni satisfactoria, y si no encuentran a nadie que intervenga para frenar esta situación, se deja pasar el tiempo y cada vez el problema se va haciendo mayor. Así, nos encontramos con dificultades en el aprendizaje cómo las analizadas en nuestra investigación. Cuando estas dificultades son detectadas en la secundaria, la tarea de intervención en ellas con el objetivo de mejorarlas resulta mucho más compleja ya que dichas DA, están muy consolidadas y para su disminución se precisa de más tiempo y trabajo. No obstante, con esfuerzo y dedicación se pueden mejorar como hemos podido comprobar.

Volviendo al tipo de errores ortográficos cometido en estos niveles, y teniendo en cuenta que los alumnos tendrían que dominar tanto la $\mathrm{ON}$ como la $\mathrm{OA}$, los resultados observados en el presente informe corroboran la conveniencia de desarrollar programas específicos para la enseñanza de la Ortografía también en estas edades, que formen parte de la práctica habitual del aula, y en los casos en los que, como el que nos ocupa, sean necesarios, deben ser instrumentos de apoyo que se impartan de forma complementaria a las materias curriculares.

\section{Referencias Bibliográficas}

Björk L. y Blomstand, I. (2000). La escritura en la enseñanza secundaria. Los procesos del pensar y del escribir. Barcelona. Grao.

Cassany, D. (1997). Describir el escribir. Barcelona. Paidos.

Cuetos, F. (1991). Psicología de la escritura. Escuela Española.

Cuetos, F., Sánchez, C. y Ramos, J. L. (1996). Evaluación de los procesos de escritura en niños de primaria. Bordón, 48 (4), 445-456.

Flower, L. y Hayes, J. (1980). Identifying the organization of writing processes. En Gregg L. W. y Steinberg, E. R.(Eds). Cognitive processes in writing. Hillsdale. LEA.

Goswami, U. y Bryant, P. (1990). Phonological skills and learning to read. Hove. LEA.

Gough, P. B., Ehri, L. C. y Treiman, R. (1992). Reading acquisition. Hillsdale. LEA.

INCE, (2000). La Evaluación del Sistema Educativo. El País, (lunes, 26-02-01), 33 Kelogg, R. (1996). A Model of working memory in writing. En Levy, M., Ransdell, S. (Eds). The science of writing Theories, Methods, individual differences and applications. Mahwah. LEA

Mesanza, J. (2000). Los alumnos de ESO son incapaces de escribir 25 palabras sin cometer faltas. El País, (lunes, 6-11-00), 40.

Morton, J. (1980). The logogen model and orthografic structure. En Frith U. (Ed). Cognitive processes in spelling. Londres. Academic Press.

Nicasio, J. (1995). Manual de dificultades de aprendizaje. Madrid. Narcea

Nicasio, J. (2001). Manual de dificultades en el aprendizaje. Madrid. Narcea.

Romero, J. (1993). Las Dificultades de aprendizaje.Valencia. Promolibro.

Romero, J. F., y González, M. J. (2001). Prácticas de Comprensión Lectora: Estrategias para el Aprendizaje. Madrid. Alianza.

Romero, J. F. (1999). Retrasos madurativos y DA. En Marchesi, A., Coll, C. y Palacios, J. (Eds). Desarrollo psicológico y ed. III. Trastornos del desarrollo y n.e.e. Madrid. Alianza.

Rueda, M. y Sánchez. E. (1996). Relación entre conocimiento fonémico y dislexia: un estudio instruccional. Cognitiva, (8) 2, 215-234. 
Sánchez, E. y Cuetos, F. (1998). Dificultades en la lectoescritura: naturaleza del problema. En González-Pienda J. A. y Núñez, J. C. (Eds). Dificultades del aprendizaje escolar. Pirámide.

Sánchez, E. (1999). El lenguaje escrito y sus dificultades: una visión integradora. En Marchesi, A., Coll, C. y Palacios, J. (Eds). Desarrollo psicológico y educación III. Madrid. Alianza.

Scardamalia, M. y Bereiter, C. (1993). Enfoques de $1^{\circ}, 2^{\circ}$ y $3^{\circ}$ orden para mejorar las estrategias cognitivas de aprendizaje de la escritura. En Beltrán, J., Bermejo.V, Prieto, M. D. y Vence, D. (Eds): Intervención psicopedagógica. Madrid. Pirámide.

Vigotsky, L. S. (1983). The prehistory of written languaje. En Martlew, M. (Ed). The psychology of written languaje: developmental and education perspectives. Chichester. John Wiley y Sons.

Fecha de recepción: 09/01/02

Fecha de revisión: 12/02/02

Fecha de aceptación: 20/05/03 